\title{
Searching Potential Sliding Surfaces in the Rock Slope Based on Crack Network Simulation
}

\author{
Yong Zhang, Dexin Nie \& Yufeng Wei \\ Chengdu University of Technology \\ Sichuan 610059, China
}

Jin Li

China Chengda Engineering Corporation, Sichuan 610041, China

\begin{abstract}
In the process of evaluating the stability of some rock slopes, the discontinuities which mainly destroy the stability of future slope can not be understood and the locations of the slope can not be determined because of lacking of the exploration data. This paper determines the combination molds and the location of potential sliding surfaces using the method of crack network simulation to do statistics about all the discontinuities, which aims to resolving the problems of determining the locations and idiographic conformations of potential sliding surfaces in the rock slop.
\end{abstract}

Keywords: Potential sliding surfaces, Network simulation, Monte-carlo

\section{Summary}

In the process of analyzing the stability of rock slope engineering, particular the mine and road projects, the large-scale exploration can not be investigated considering the factors of manpower and material resources. Therefore, how to obtain the combination molds of the interior discontinuities of slope and how to estimate the conformation of potential sliding surfaces are the keys to evaluate the stability of slope accurately.

In the geologic investigation of slop engineering, the discontinuity parameters are acquired by the practical survey, using the method of simply geometric projection to determine the features of slope which do not consider the complex configuration of jointed rock mass and the internal mechanism about destroying the combination of discontinuities (Jia, Zhixin \& Wang Xiaogang. 2001)(Zhang X. 1989). With the development of computer technology and the continual integration of different subjects, the probability models of geometric parameters used to acquire the sample data of geometric parameter statistics about different configuration surfaces can be obtained through the theory of probability statistics on the basis of data recorded in the field. Then the combination molds of slop discontinuities can be obtained and the features and locations of the potential sliding surfaces of slope can be simulated using the network simulation method.

\section{Theoretical basis}

With the development of slop engineering, it is found that an abundance of disorderly and unsystematic joint surfaces (mainly referring to the grade IV, V discontinuities) distribute randomly in the rocks, whose features are reflected by the geometric parameters (spacing interval, tendency, dip, trace length etc) obeying some statistical law in the view of statistics. The probability statistic models are built using the abundance of sample data acquired in the practical survey. The models reflected in the computers form the crack network simulation figures having the same statistic characteristics and token discontinuities distributing features, which make use of the Monte-carlo method(Jia, Zhixin \& Wang, Xiaogang. 2001)(Hudson J A \& Priest S D. 1979).

The Monte-carlo method is also named random simulation method or statistic testing method, which is the method to seek random variables making use of uniformly random numbers according to the known distributing functions. In fact, the Monte-carlo simulation is the contradictorily process of practical sampling statistics. The practical surveying statistics seek for all kinds of geometric parameter distributing functions representing cracks according to the distributing forms of cracks in the rock. However, the process of computer simulation seeks for the crack network figures obeying to these distributing laws according to the distributing functions of crack geometric parameters obtained by the statistics. In the crack network simulation, for the purpose of educing the random variables of distributing functions obtained in the practical survey, one should obtain the uniformly distributed random numbers through the computers firstly, then sample randomly among the numbers to seek for the practically distributing random numbers according to the known practically distributing functions (Wei, An. 1995)(Pan, Bietong. 1984)(Xu, Zhongji. 1985). 


\section{Engineering examples}

\subsection{Basic geological condition}

The mudstone mine in the Duyan city locates in the junctional region between basin and coteau, which belongs to the low mountain landform of structural erosion, and the general terrain of the mine is higher in north and lower in south. The relative height difference in the mine is 215 meters. The south of the mine represents the gentle slope morphology and steeper dips form because of the east-west direction of the mine cut by rivers.

The outcrop in the mine is the middle segment of the Triassic Xujia river series $\left(\mathrm{T}_{3}{ }^{\mathrm{x} 2}\right)$ and the stratum covered by the residual deposit of quaternary slop $\left(\mathrm{Q}_{4}{ }^{\mathrm{edl}}\right)$. The attitude of rocks is $350^{\circ} \angle 25$. The exploited ores in the mine mainly are the mudstones and sandstones of the Xujia river series.

The mine locates in the south limb of syncline belonging to the Lanbandeng, namely the Lanbandeng of white stone flying peak, and the configuration is mainly NNE-SSW. The faults in the mine are agenesis and the cracks are dense and the rocks are broken.

With the exploitation of the mine, the surface may form the stepladder terrain with the height of 5 meters and the width of 5 meters and the slop dip of about $60^{\circ}$. The designable fixed number of year for exploiting the mine is 50 years, so the slope are the forever ones.

In the process of continually digging, the slope of mine form gradually, so the stability of slope has to be evaluated in the process.

\subsection{The parameter statistics of crack network simulation}

Many sections are disposed in the mine and the abundance of discontinuity parameter data has to be surveyed in the vicinity of the sections for the probability statistics. This paper works out the polar iso-density map (figure 1) applying the mapping method of stereographic pole projection. Then the configuration surfaces are grouped to obtain the sample data of geometric parameter statistic of configuration surfaces for each group. The next step is that the discontinuities of each group are analyzed statistically and curve simulated according to the geometric parameters of discontinuities, so the according parametric probability models are obtained.(Table 1)

\subsection{Crack network simulation and searching potential sliding surfaces}

The figure of rock discontinuity network simulation is obtained using the Monte-carlo method on the basis of the statistic features of the above discontinuities (shown in figure 2). It is found in the figure the features of discontinuities that the network simulation figure shows accord to the results of the polar iso-density map. At the same time, the dips in the discontinuities are generally steep, so the connectivity rate of the cracks in the vertical direction is higher. However, the effective connectivity rate of the cracks in the horizontal direction is not so high but connected by the rock bridge.

The network simulation results of discontinuities are embedded in the slope dug in the futures to obtain the combination figure of discontinuities of the slopes. It is found in figure 3 that many kinds of incision formats of the combination between every discontinuity and dug surface. The combination of discontinuities (1) (2) is typical or the most adverse format in the view of the scale of sliding mass, the length of potential sliding surface and the degree of steep dip. It can be judged that the combination of discontinuities (1) (2) and (3) (4) is the most adverse format by comparing different combination kinds of discontinuities, which may form the potential sliding surface of the future slope, according to which the stability of slope can be evaluated.

\section{Conclusions}

This paper proposes the method of crack network simulation based on Monte-carlo researches the method of searching the potential sliding surface in the rock slope on the basis of the connection of the discontinuities statistics and the random simulation. For researching the combinatorial problems of discontinuities in the interior of the rock slope, one adopts the method from the surface to the centre using the statistical law of exterior cracks to simulate the combination of crack network within the slope, which determines the forms and locations of the potential sliding surfaces in the slope to provide the theoretical basis for analyzing the stability of slope and possess the important reference significance for the digging and treatment in the latter slope engineering.

\section{References}

Hudson J A \& Priest S D. (1979). Discontinuities and rock mass geometry. Intermational Journal for Rock Mechanics and Mining Science. 16(2):339-362.

Jia, Zhixin \& Wang, Xiaogang. (2001). Persistence computation and application of jointed rock mass including large identified discontinuities. Chinese Journal of Rock Mechanics and Engineering. 2001,21(4):457-461. 
Pan, Bietong. (1984). The foreign research status of probability models for discontinuity network of a rock slope. Geological Science and Technology Information. 9(2):45-50.

Wei, An. (1995). A Computerized Simulation of the Crack Network in Rock Mass and Its Applications. Journal of Southwest Jiaotong University. 30(2):200-205.

Xu, Zhongji. (1985). The Monte-carlo Method. Shanghai: Shanghai Scientific Publisher.

Zhang X. (1989). A two dimensional model of en-echelon jointed rock masses with mulit-discontinuity geometry parameters. Rock Mechanics and Rock Engineering. 22(3):231-142.

Zhang, Yongrong, Yu, Hongming \& Yin, Kunlong etc. (2000). Analog and Analysis of Discontinuity Network Of A Rock Slope in Jiangxi. Geological Science and Technology Information. 19(1):85-88.

Table 1. Parameters of Discontinuity in section I

\begin{tabular}{|c|c|c|c|}
\hline Amount of discontinuity groups & \multicolumn{3}{|c|}{ Three } \\
\hline Number of discontinuity groups & first & second & third \\
\hline Type of intervals distribution & normal & normal & lognormal \\
\hline Average of intervals & 0.12 & 0.13 & 0.29 \\
\hline Variance of intervals & 0.02 & 0.07 & 0.22 \\
\hline Type of tendencies distribution & normal & normal & Negative exponetial \\
\hline Average of tendencies & 93.00 & 184.00 & 274.33 \\
\hline Variance of tendencies & 7.60 & 26.93 & 14.74 \\
\hline Type of dips distribution & normal & normal & Negative exponetial \\
\hline Average of dips & 83.00 & 64.48 & 79.00 \\
\hline Variance of dips & 4.53 & 10.54 & 1.00 \\
\hline Type of trace lengths distribution & Normal & normal & Negative exponetial \\
\hline Average of trace lengths & 0.80 & 0.46 & 0.73 \\
\hline Variance of trace lengths & 0.85 & 0.20 & 0.28 \\
\hline
\end{tabular}

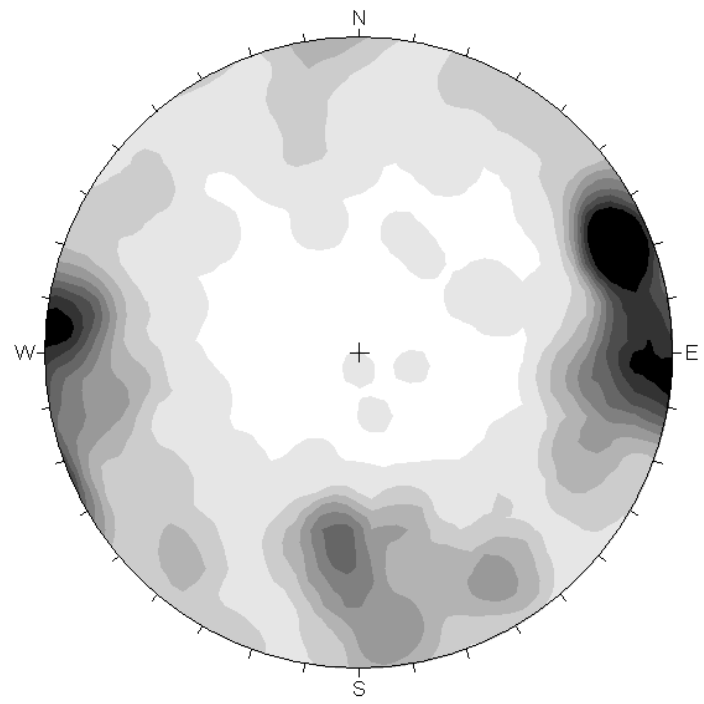

Figure 1. A graph of isopycnic pole discontinuity in section I 


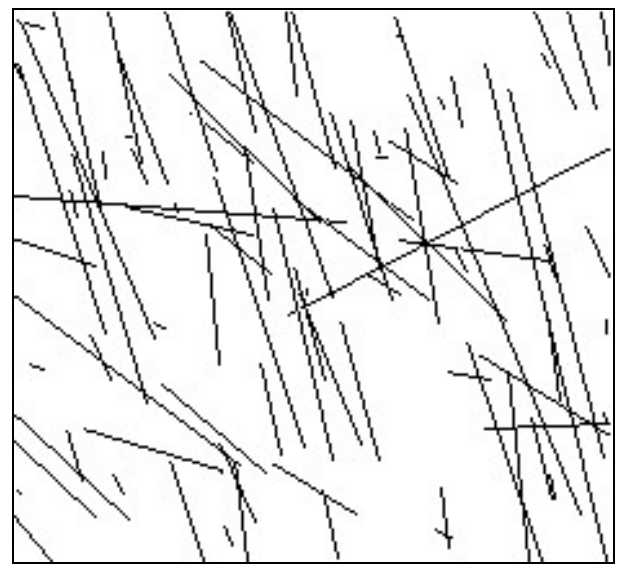

Figure 2. Network simulation of discontinuity

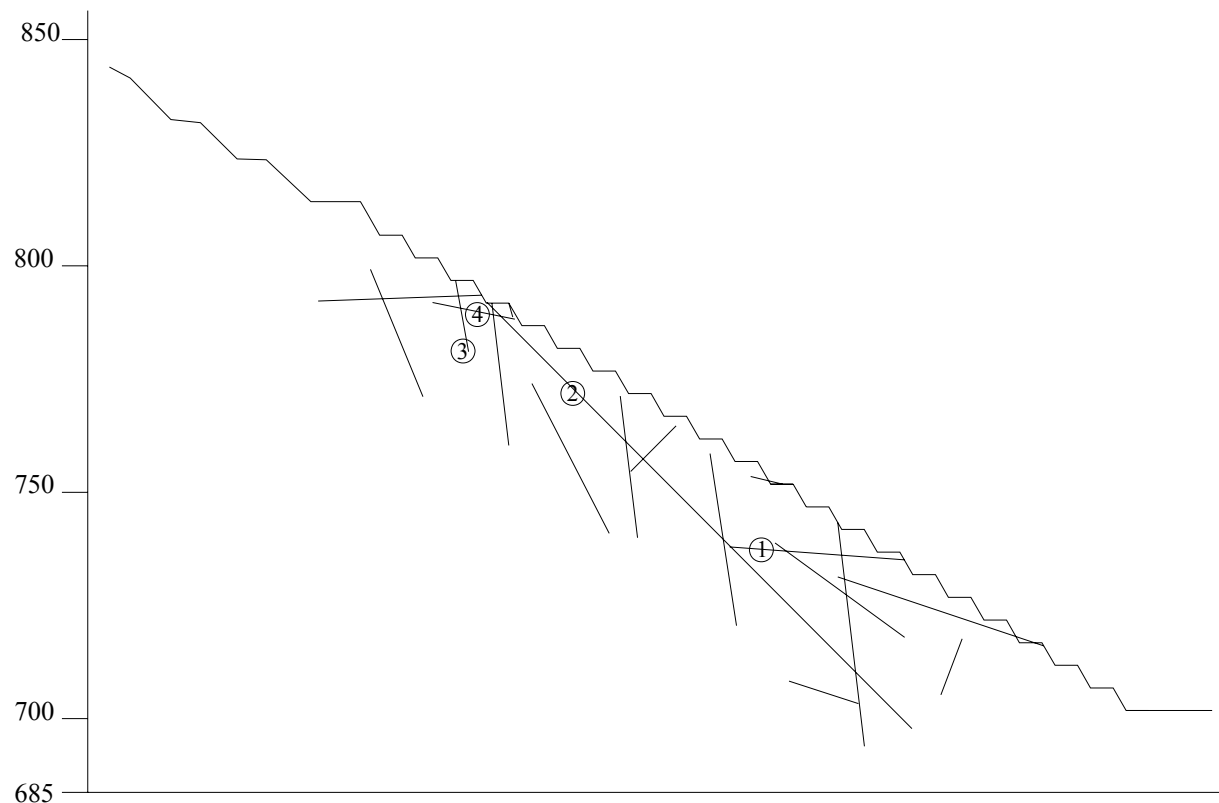

Figure 3. Combination of discontinuity in section I 